\title{
Linear Medullary Pericanal Lesion with Longitudinal Myelitis can be Diagnostic of Neuromyelitis Optica
}

\author{
Yuji Tomizawa ${ }^{1}$, Jiro Fukae ${ }^{1}$, Kazuyuki Noda ${ }^{1}$, Kenji Fujishima ${ }^{1}$, Kazumasa Yokoyama ${ }^{2}$, \\ Nobutaka Hattori ${ }^{2}$, Toshiyuki Takahashi ${ }^{3}$ and Yasuyuki Okuma ${ }^{1}$
}

Key words: NMO, medullary lesion, hiccup, long cord lesion

(Inter Med 48: 175-176, 2009)

(DOI: 10.2169/internalmedicine.48.1719)
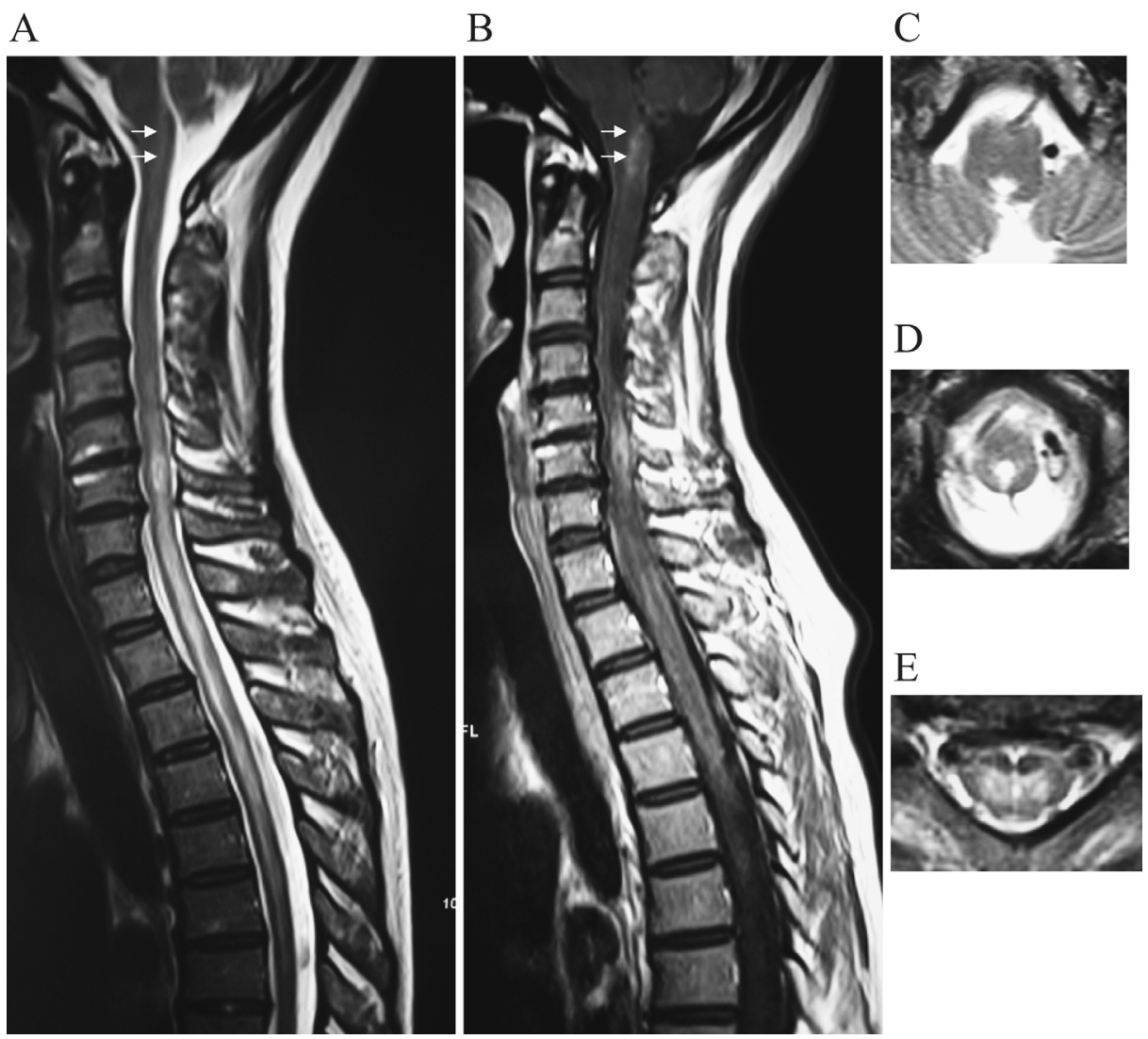

Picture 1. T2-weighted sagittal spinal MR image shows a high signal intensity long cord lesion extending from the $\mathrm{C} 3$ to the T6 level (A). Linear faint T2 high intensity signals were also demonstrated in the medulla oblongata (arrows) (A). Both the spinal cord lesion and medullary lesion were contrast-enhanced in sagittal T1-weighted images (arrows) (B). T2-weighted brainstem MR images show pericanal lesions seen in the medulla oblongata $(C, D)$. T2-weighted axial image of the C6 level shows centrally located high signal intensities within the spinal cord (E).

\footnotetext{
${ }^{1}$ Department of Neurology, Juntendo University Shizuoka Hospital, Shizuoka, ${ }^{2}$ Department of Neurology, Juntendo University School of Medicine, Tokyo and ${ }^{3}$ Department of Neurology, Tohoku University Graduate School of Medicine, Sendai Received for publication September 27, 2008; Accepted for publication September 30, 2008 Correspondence to Dr. Yasuyuki Okuma, sgz02202@nifty.ne.jp
} 
deep tendon reflexes in all extremities. She complained of decreased sensation below the C5 spinal level. Although sicca symptoms were not observed, the results of laboratory data and a lip biopsy fulfilled the criteria of Sjögren's syndrome. The serum anti-aquaporin-4 (AQP4) antibody titre (1) was $256 \times$. A lumbar puncture revealed pleocytosis of 368 cells $/ \mu \mathrm{L}$ (357 mononuclear cells) with an increased protein level of $90 \mathrm{mg} / \mathrm{dL}$. T2-weighted MRI showed a highintensity long cord lesion extending from the C3 to the T6 level and a linear pericanal lesion in the medulla oblongata (Picture 1). These lesions were gadolinium enhanced. She was diagnosed as having neuromyelitis optica (NMO) spectrum disease (2) associated with Sjögren's syndrome and was successfully treated with IV methylprednisolone with subsequent oral prednisolone.

Misu and colleagues (3) reported six patients with recur- rent NMO, who exhibited intractable hiccup with linear medullary lesions as seen in our patient. The present case further suggests that NMO spectrum disorder may be diagnosed in patients having longitudinal myelitis and characteristic medullary lesions, even without optic neuritis or without recurrent episodes. Because NMO requires long-term treatment with immunosuppressants, correct diagnosis should be made by measuring the anti-AQP4 antibody/ NMO-IgG in such patients. Another interesting finding was that our patient had Sjögren's syndrome. Since longitudinal myelitis has been reported in Sjögren's syndrome (4), we speculate that some patients previously reported as having Sjögren myelitis may also have had NMO spectrum disorder. We emphasize that awareness of these MRI lesions will lead to the successful management of this occasionally devastating disorder.

\section{References}

1. Takahashi T, Fujihara K, Nakashima I, et al. Anti-aquaporin-4 antibody is involved in the pathogenesis of NMO: a study on antibody titre. Brain 130: 1235-1243, 2007.

2. Weinshenker BG. Neuromyelitis optica is distinct from multiple sclerosis. Arch Neurol 64: 899-901, 2007.

3. Misu T, Fujihara K, Nakashima I, Sato S, Itoyama Y. Intractable hiccup and nausea with periaqueductal lesions in neuromyelitis optica. Neurology 62: 1479-1482, 2005.

4. Yamamoto T, Ito S, Hattori T. Acute longitudinal myelitis as the initial manifestation of Sjögren's syndrome. J Neurol Neurosurg Psychiatry 77: 780, 2006.

(C) 2009 The Japanese Society of Internal Medicine http://www.naika.or.jp/imindex.html 